Cuadernos I. Geográfica

25

pp. $85-110$

Logroño

1999

\title{
FACTORES HÍDRICOS Y EROSIONABILIDAD EN EL GRADIENTE EDAFOCLIMÁTICO DEPRESIÓN DEL EBRO / PIRINEO CENTRAL
}

\section{MANUEL SEEGER ${ }^{1}$ CHRISTIAN FREVEL ${ }^{2}$}

RESUMEN: Se han estudiado los suelos en cinco áreas de muestreo a lo largo de un transecto entre la Depresión Central del Ebro y el Pirineo Central español con el fin de detectar un posible gradiente en cuanto a la erosionabilidad y a diferentes parámetros hidricos del suelo.

El estudio de los suelos de este transecto muestra ciertas similitudes, pudiendo observarse una ligera tendencia a la disminución de la erosionabilidad desde la Depresión del Ebro basta el Pirineo Central. La erosionabilidad del material matricial del suelo es, de todas formas, alta en todas las áreas estudiadas, siendo moderada de forma significativa por la pedregosidad en las zonas situadas fuera de la Depresión central del Ebro. De los resultados obtenidos se deduce que las diferencias en la producción de sedimento dependen primordialmente de la cobertura vegetal y de la erosividad de las precipitaciones.

Las condiciones bidricas del suelo muestra ser dentro de cada área estudiada mucho más variable que a lo largo del transecto. Se han llegado a estimar en zonas erosionadas valores de capacidad de retención de agua disponible extremadamente bajos. De igual forma, todos los suelos muestran conductividades bidráulicas muy bajas en condiciones no saturadas. En consecuencia, las precipitaciones generan una elevada escorrentia, que a su vez erosiona los suelos e impide su recarga bidrica.

1. Institut für Physische Geographie, Albert Ludwigs Universität Freiburg (Alemania).

E-mail: M.SEEGER@teleline.es

2. Institut für Geographie und Geoökologie I, Universität Karlsruhe (TH), Alemania. 
MANUEL SEEGER - CHRISTIAN FREVEL

Del conjunto de características físicas de los suelos entre la Depresión Central del Ebro y el Pirineo Central se puede deducir que las capacidades bidráulicas tenderán a deteriorarse a causa de la alta erosividad de la matriz edáfica. De este modo, las condiciones para la recuperación de la cubierta vegetal tras el los cambios de uso del suelo serán cada vez más adversas.

ZUSAMMENFASSUNG. Entlang eines Transekts zwischen dem Inneren Ebrobecken und den Zentralpyrenäen sind die Böden von fünf Testgebieten untersucbt worden, um mögliche Gradienten in der Erodierbarkeit und bodenbydraulischen Parametern entlang des Transektes herauszuarbeiten.

Es zeigt sich, dass die Erodierbarkeit der Böden aller Testgebiete boch ist und lediglich eine schwache Abnabmetendenz vom Inneren Ebrobecken bin zum Pyrenäenhauptkamm aufweist. In den Gebieten ausserbalb des Ebrobeckens wird die Erosionsanfälligkeit der Bodenmatrix stark vom Bodenskelett reduziert.

Die bodenbydrauliscben Bedingungen zeigen innerbalb der Gebiete eine größere Variabilität wie entlang des Transektes. In stark erodierten Bereichen sind extrem niedrige effektive Feldkapazitäten festgestellt worden. Zusätzlich weisen alle Böden im allgemeinen extrem niedrige ungesättigte Wasserleitfähigkeiten auf. Dies fübrt zu einer raschen Oberflächenabflußbildung, was gleichzeitig eine verstärkte Erosion und eine unvollständige Auffüllung des Wasserspeichers bewirkt.

Es läßt sich, aufgrund der bodenpbysikalischen Daten, eine zunebmende Degradierung der bodenbydraulischen Parameter vorausseben. Dies fübrt zu ungünstigen Bedingungen für die Wiederberstellung der Pflanzendecke nach dem Wandel der Landnutzung.

ABSTRACT. The soils of 5 test areas along a transect between the Inner Ebro Basin and the Central Pyrenees have been studied, in order to detect possible gradients in soil erodibility and bydraulic properties.

All studied soils show similar values of erodibility, with a slight trend to decrease from the Ebro Basin to the Pyrenees. In all areas, except the one in the Inner Ebro Depression, the soil stoniness reduces considerably the erodibility of the soils.

Soil bydraulic conditions show a bigger variability inside the test areas than along the transect. Strongly eroded areas show very low 
FACTORES HÍDRICOS Y EROSIONABILIDAD EN EL GRADIENTE EDAFOCLIMÁTICO

values of available water. In addition, the soils of all test areas show very low water conductivities under unsaturated conditions. This leads to a bigh runoff, resulting in soil erosion and in incomplete filling of the soil water storing capacity.

Soil pbysical data of the studied areas show that the soil bydraulic conditions will degrade with time. This leads to unfavorable conditions for restoring vegetation after land use change.

Palabras clave: Erosionabilidad de suelos, Parámetros físicos de suelos, Transecto, Depresión del Ebro, Pirineos.

Schlagwörter: Erodierbarkeit, Bodenphysikalische Parameter, Transekt, EbroDepression, Pyrenäen.

Key words: Erodibility, Physical soil properties, Transect, Ebro-Depression, Pyrenees.

\section{Introducción}

Las alteraciones sufridas por el paisaje del norte de Aragón han sido causadas en su mayor parte por la acción humana, que en los últimos 6000 años ha creado una población estable y creciente en numerosas zonas entre el Valle del Ebro y el Pirineo Central (García-Ruiz \& Valero, 1996).

Durante las ultimas décadas se ha producido, en cambio, una disminución notable de la densidad de población, hasta la casi completa despoblación de comarcas enteras, con el consecuente abandono y la extensificación de los usos agrícolas. Así, la superficie cultivada en el Pirineo ha descendido de aproximadamente el $28 \%$ a mediados del siglo pasado hasta menos de un $3 \%$ en la actualidad (García-Ruiz \& Lasanta, 1990; García-Ruiz et al., 1991). Estos cambios se irán acentuando más como consecuencia de la Política Agraria Comunitaria, que apoya el abandono de tierras marginales de baja productividad, subvencionando al mismo tiempo la ganadería ovina. De todas formas, la dinámica del abandono se traslada hacia las tierras bajas, en la Depresión Central del Ebro, siendo esta zona la más afectada en la actualidad por los abandonos de cultivos.

El área de estudio entre el Pirineo Central y la Depresión del Ebro ofrece un gradiente climático excepcional en el conjunto de Europa occidental. De este modo es posible estudiar en una distancia de tan solo varias decenas de kilómetros la evolución del paisaje bajo condiciones de impacto humano parecidas.

La respuesta geomorfológica y ecológica a estas alteraciones depende de varios factores, entre los que destaca la evolución de la cubierta vegetal. Esta a su vez depende del uso actual del territorio y de las condiciones edáficas resultantes del uso anterior y de la evolución del suelo en el periodo transcurrido. 
Diferentes aspectos del abandono de tierras en el norte de Aragón han sido estudiados por varios autores, especialmente la evolución hidrológica y geomorfológica (Garcia-Ruiz et al., 1996), la erosión y degradación de los suelos y la evolución del paisaje (García Ruiz et al., 1994; Schad et al., 1992; Cernuska et al., 1996; Ries et al., 1997; Marzolff 1998) al igual que su dependencia de la gestión del paisaje (Molinillo et al., 1997).

El presente estudio se centra en las condiciones edáficas de las áreas afectadas por la extensificación del aprovechamiento agrícola. Por un lado se han estudiado las características relacionadas con la disponibilidad de los recursos hídricos de los suelos a lo largo del transecto, ya que recientes resultados indican que los nutrientes no han de ser el factor limitante de una recolonización vegetal de los suelos (Seeger, 1997); por otro lado se han analizado los factores más relacionados con la erosionabilidad edáfica. Se trata, en definitiva, de averiguar de qué forma varían aspectos geoecológicos fundamentales, en función sobre todo del gradiente climático que se establece entre el centro de la Depresión del Ebro y el Pirineo Central.

\section{Areas de estudio}

Se han estudiado los suelos de las cinco áreas incluidas en el proyecto EPRODESERT (Ries et al., 1997) entre las Sierras Interiores del Pirineo Central y la Depresión Central del Ebro (Figura 1):

1. Aísa: El área seleccionada en el valle de Aísa se encuentra en el límite septentrional de las Sierras Interiores en la zona dominada por el Flysch Eoceno Surpirenaico. Se encuentra a $1700 \mathrm{~m}$ s.n.m., orientada hacia el oeste y con pendientes alrededor de los $25^{\circ}$. Está situada por encima del límite forestal actual, con predominio de pastos subalpinos; sin embargo, la vegetación potencial se caracterizaría por la presencia de bosques de Pinus nigra (Ibarra \& Martínez Ferrer, 1995). El clima es claramente húmedo, con un excedente hídrico anual superior a los $500 \mathrm{~mm} /$ año (Cuadrat, 1995).

2. Cuenca de Arnás (Valle de Borau): La cuenca de Arnás se localiza igualmente en el flysch eoceno. El área de muestreo se encuentra en un coluvión aterrazado en la solana del valle a $940 \mathrm{~m}$ s.n.m.. La vegetacón potencial estaría compuesta por bosques de Quercus faginea, Quercus pubescens y Pinus silvestris (Ibarra \& Martínez Ferrer, 1995), aunque la planta dominante en la actualidad es, al menos en la zona inferior de la ladera, Genista scorpius. Las zonas intensamente pastoreadas están cubiertas de hemicriptófitos y terófitos de escasa cobertura. El balance hídrico anual muestra un superávit de unos $350 \mathrm{~mm}$ /año (Cuadrat, 1995).

3. Bentué de Rasal: Se encuentra en la zona central de las Sierras Exteriores, en un valle abierto en las margas azules eocenas, fácilmente erosionables. La 


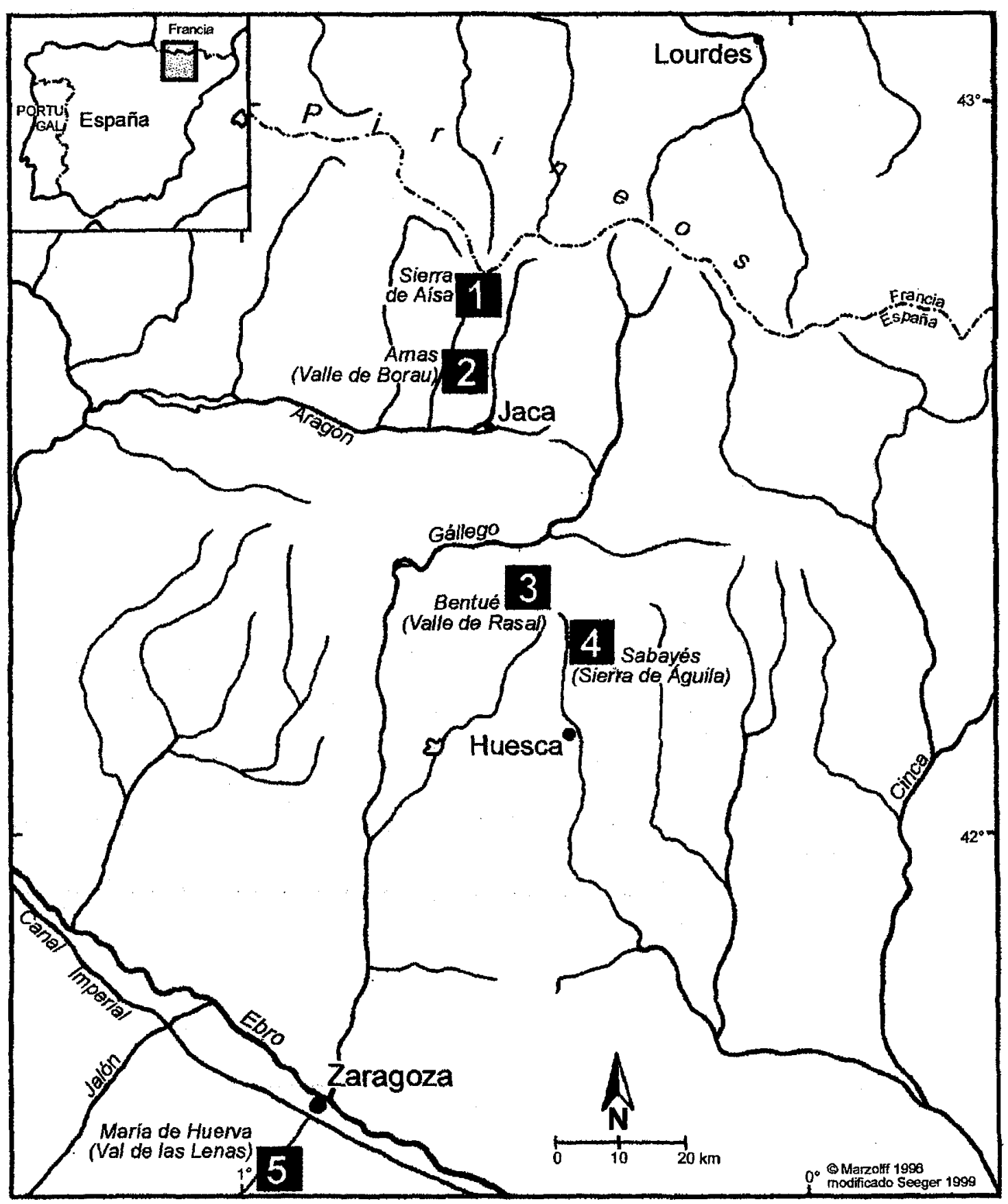

Figura 1: Situación de la áreas estudiadas. Se muestra la red fluvial y las poblaciones más importantes.

Abb.1: Lage der Arbeitsgebiete. Es sind das Gewässernetz und die wichtigsten Ortschaften der Region abgebildet.

vegetación potencial es parecida a la indicada en el área descrita anteriormente (Ibarra \& Martínez Ferrer 1995), aunque la vegetación actualmente dominante se caracteriza por un mosaico compuesto por Genista scorpius, 
MANUEL SEEGER - CHRISTIAN FREVEL

Buxus sempervirens y Thymus vulgaris. El clima es subhúmedo, con un ligero superávit hídrico de aproximadamente $100 \mathrm{~mm} /$ año (Cuadrat 1995).

4. Sabayés: El área de trabajo se sitúa en antiguas terrazas de viñedos, sobre de un derrubio de ladera compuesto por margas y calizas, en el borde norte de la Depresión del Ebro. Actualmente, la vegetación se caracteriza por formaciones dominadas por Genista scorpius, aunque la vegetación potencial se compondría de matorral y bosques de Quercus coccifera, en las partes inferiores, y Quercus pubescens, en las superiores (Ibarra \& Martínez Ferrer 1995). La zona se encuentra entre el llano semiárido y el Prepirineo subhúmedo, con un déficit hídrico medio de unos $100 \mathrm{~mm} /$ año (Cuadrat 1995).

5. Maria de Huerva: Se localiza en el centro de la Depresión del Ebro, área dominada por sedimentos terciarios continentales: margas, yesos y margas yesíferas intercaladas, y coluviones holocenos. La vegetación es típica esteparia de influencia térmica (Suárez Cardona et al. 1989), caracterizada por formaciones dominadas por Lygeum spartum de escasa cobertura. El clima, semiárido, muestra una gran variabilidad intra e interanual de las precipitaciones. El déficit hídrico medio es de aprox. $450 \mathrm{~mm} /$ año (Cuadrat, 1995).

Descripciones más detalladas de estas áreas de estudio se encuentran en Ries, 1995, Molinillo et al., 1997, Seeger 1997, Ries et al., 1997.

\section{Métodología}

\subsection{Análisis de suelos}

Se han realizado 34 perfiles de suelo, cuya caracterización se efectuó según la Bodenkundliche Kartieranleitung (KA 4) (AG Boden, 1994). Los puntos de muestreo se escogieron tras una primera aproximación de campo y de fotografías aéreas tomadas dentro del proyecto EPRODESERT (Marzolff, 1998).

Para clasificación de los suelos se han seguido las normas propuestas en la Soil Taxonomy (USDA, 1998), tanto para la nomenclatura como para los análisis. Únicamente la granulometría fue determinada según las normas DIN 19683 (1973), que implica unos tamaños de granos algo diferentes a los establecidos por la USDA.

\subsection{Determinación de la erosionabilidad}

Para la estimación de la erosión se ha utilizado la Universal soil Loss Equation (USLE) de Wischmeier \& Smith (1978), en una versión modificada por Schwertmann et al. (1987), la denominada Allgemeine Bodenabtragsgleichung (ABAG). El factor $\mathrm{K}$ se ha calculado siguiendo a estos autores.

Aun teniendo en cuenta las limitaciones de la USLE se ha considerado válido realizar este estudio, esencialmente para poder comparar los suelos analizados. 


\section{FACTORES HÍDRICOS Y EROSIONABILIDAD EN EL GRADIENTE EDAFOCLIMÁTICO}

Por eso mismo se centra la mirada en el cálculo del factor $\mathrm{K}$, que de este modo refleja las condiciones edáficas, descartando variaciones estacionales y eventuales de parámetros edáficos, que pueden tener una influencia determinante en la erosionabilidad del suelo (véanse Young et al., 1990; Fohrer, 1995; Gerlinger, 1997).

\subsection{Determinación de los parámetros hidráulicos}

Los parámetros hidraúlicos del suelo han sido determinados en laboratorio. Para ello se han analizado muestras no perturbadas de suelo y se han determinado las curvas pF y la conductividad hidráulica de la mayoría de los horizontes de los perfiles más significativos de cada área estudiada. Los valores obtenidos han sido corregidos conforme a las cantidades de yeso encontradas en los suelos de María de Huerva, o de las cantidades de piedras en las otras áreas de trabajo.

La capacidad de retención de agua disponible (CRAD) se ha calculado segun los criterios de la KA 4 (AG Boden 1996), utilizando para ello el punto de pF 1,8 como capacidad de campo.

Las carácteristicas hidráulicas de los suelos se han parametrizado según van Genuchten (1980), y partiendo de los valores calculados se ha estimado la conductividad hidráulica del suelo no saturado (Ku), utilizando el modelo van Genuchten-Mualem (van Genuchten, 1980). Se incluyen, a modo de ejemplo, los valores de la conductividad hidráulica del suelo no saturado calculados de un perfil por área estudiada.

\subsection{Valoración de los resultados analíticos}

La erosibilidad, la densidad aparente, los poros gruesos, la capacidad de campo y la CRAD han sido valorados segun AG Boden (1994); esta última se ha valorado en el caso de los suelos de María de Huerva y Sabayés segun la USDA (1983, en: Porta et al. 1999). La clasificación de la conductividad hidráulica se hizo según la FAO (1963, en: Porta et al. 1999: 351). Estos ultimos valores se han obtenido con fines comparativos entre los diferentes suelos, ya que los resultados difieren normalmente de forma considerable de los medidos en el campo.

\section{Resultados}

Han sido clasificados cinco órdenes de suelos, según la Soil Taxonomy (USDA 1997), en las cinco áreas estudiadas. Inceptisoles y Entisoles, caracterizados por una diferenciación de horizontes escasa o inexistente, son dominantes en todas las zonas excepto la Depresión Central del Ebro. En Arnás, Bentué de Rasal y Aísa tambien se han cartografiado Alfisoles. Además, en Arnás se ha descrito un Mollisol. En María de Huerva, en la Depresión del Ebro, los suelos pertenecen a la orden de los Aridisoles (véase Tabla 1). 
MANUEL SEEGER - CHRISTIAN FREVEL

Tabla 1: Relación de los suelos cartografiados en las áreas estudiadas.

Tabelle 1: Übersicht über die Klassifikation der untersuchten Profile

\begin{tabular}{|c|c|c|c|c|c|}
\hline Orden & Maria de Huerva & Sabayés & Bentué de Rasal & Arnas & Aisa \\
\hline Aridisols & $\begin{array}{c}\text { Lithic/Leptic/Typic } \\
\text { Haplogypsids }\end{array}$ & - & - & - & - \\
\hline Entisols & Xeric Torriorthent & $\begin{array}{c}\text { Oxyaquic } \\
\text { Xerorthent }\end{array}$ & $\begin{array}{c}\text { Lithic Xerorthents } \\
\text { Lithic/Typic Ustorthents }\end{array}$ & $\begin{array}{c}\text { Lithic/Typic } \\
\text { Ustorthents }\end{array}$ & $\begin{array}{c}\text { Lithic } \\
\text { Cryorthent }\end{array}$ \\
\hline Inceptisols & - & $\begin{array}{c}\text { Haplic } \\
\text { Calcixerept }\end{array}$ & $\begin{array}{c}\text { Calcic/Typic Haplustept } \\
\text { Haplustept } \\
\text { Aquic Calciustept } \\
\text { Typic/Aridic }\end{array}$ & $\begin{array}{c}\text { Mollic } \\
\text { Dystrochryept }\end{array}$ \\
\hline Alfisols & - & - & Inceptic Haplustalfs & $\begin{array}{c}\text { Oxyaquic Vertic } \\
\text { Haplustalf }\end{array}$ & $\begin{array}{c}\text { Aquic } \\
\text { Cryoboralf }\end{array}$ \\
\hline Mollisols & - & - & - & Lithic Rendoll & \\
\hline
\end{tabular}

\subsection{Maria de Huerva}

En María de Huerva los suelos cartografiados que ocupan mayor superficie pertenecen al grupo de los Haplogypsids, del orden de los Aridisols, que en las laderas expuestas al norte muestran variantes de menor espesor. En las laderas expuestas al sur y al oeste, de mayor pendiente y menor cubierta vegetal, se han descrito, a causa de la alta actividad morfológica reinante, suelos de escaso desarrollo, los xeric Torriorthents, del Orden de los Entisoles.

Entre los Haplogypsids se diferencian dos subgrupos: los Typic Haplogypsids, se encuentran en zonas menos perturbadas y pueden reconocerse bien por una densa costra formada por líquenes y una fuerte actividad de lombrices. Los Leptic Haplogypsids, de mayor contenido en yesos en el epipedon, se caracterizan además por el desarrollo de una costra edáfica gruesa. Estos dos tipos de suelo pueden encontrarse tanto en las zonas abandonadas hace ya varias décadas como en las abandonadas hace menos de siete años.

\subsubsection{Valores de erosionabilidad}

La erosionabilidad de los suelos de María de Huerva es en general muy alta (Tabla 2), a causa del alto contenido en limos. Solamente los suelos menos perturbados y con una alta actividad de lombrices, que hacen disminuir el contenido en yesos en la superficie e incorpora materia orgánica al suelo, reducen los valores de erosionabilidad. El perfil P21, situado ya fuera del área estudiada en un campo cultivado, muestra también un valor alto a causa del alto contenido de arena. 
FACTORES HÍDRICOS Y EROSIONABILIDAD EN EL GRADIENTE EDAFOCLIMÁTICO

Tabla 2: Factores K calculados para el epipedon de los suelos de la zona de María de Huerva.

Tabelle 2: Berechnete K-Faktoren für die Oberböden im Untersuchungsgebiet Maria de Huerva.

\begin{tabular}{|c|l|c|c|c|c|l|}
\hline \multicolumn{2}{|c|}{ Perfil } & Arena & \multicolumn{1}{|c|}{ Limo } & Arcilla & Factor K & Valoración \\
\cline { 1 - 5 } Nr. & Suelo & \multicolumn{3}{|c|}{$\%$} & & \\
\hline P1 & Leptic Haplogypsid & 17,1 & 75,5 & 7,5 & 0,72 & Muy alto \\
\hline P2 & Leptic Haplogypsid & 17,2 & 69,4 & 13,3 & 0,67 & Muy alto \\
\hline P5 & Leptic Haplogypsid & 4,2 & 70,7 & 25,1 & 0,51 & Muy alto \\
\hline P16- AyC & \multirow{2}{*}{ Lithic Haplogypsid } & 3,7 & 71,8 & 24,5 & 0,56 & Muy alto \\
\cline { 1 - 6 } P16- Cry(A) & & 6,4 & 89,2 & 4,2 & 0,76 & Muy alto \\
\hline P17 & Xeric Torriorthent & 1,4 & 69,2 & 29,4 & 0,58 & Muy alto \\
\hline P19 & Typic Haplogypsid & 6,1 & 58,7 & 35,2 & 0,34 & Alto \\
\hline P20 & Typic Haplogypsid & 7,3 & 59,8 & 33 & 0,41 & Alto \\
\hline P21 & Leptic Haplogypsid & 15,8 & 56,8 & 27,3 & 0,47 & Alto \\
\hline
\end{tabular}

\subsubsection{Parámetros hidráulicos}

El Leptic Haplogypsid del campo abandonado hace tan sólo unos siete años muestra en los primeros $15 \mathrm{~cm}$ una conductividad hidráulica y una porosidad considerablemente menores que en los $15 \mathrm{~cm}$ subyacentes. Por debajo de los $30 \mathrm{~cm}$ se encuentran los valores más bajos medidos en el perfil de estos dos parámetros (Tabla 3). La conductividad hidráulica no saturada del epipedon disminuye de forma doble logarítmica a medida que se reduce la humedad (Fig. 2). Con valores de $\mathrm{pF}<1,8$ se calcularon valores $\mathrm{Ku}<0,1 \mathrm{~mm} \mathrm{~h}^{-1}$. Por el contrario, en el Horizonte $2 \mathrm{C}$ se mantiene una conductividad hidráulica mayor de $0,1 \mathrm{~mm} \mathrm{~h}^{-1}$ con tensiones menores a 1,8. La distribución de los valores de conductividad hidráulica reflejan bien la influencia de los procesos de encostramiento y de la labor con maquinaria pesada (Porta et al. 1998). En esta zona también se encuentran suelos con valores de yesos muy inferiores a los de los Leptic Gypsisols que igualmente tienen tendencia a formar costras, pero manteniendo gran parte de los poros gruesos y medianos, lo cual conlleva a una conductividad hidráulica saturada alta respecto a los suelos antes mencionados. Sin embargo, la conductividad no saturada disminuye asimismo rápidamente con la desecación del suelo.

En el campo abandonado hace varias décadas se puede observar un volumen total de poros aproximadamente constante en todo el perfil de este Leptic Haplogypsid (Tabla 4). El volumen de poros gruesos disminuye con la profundi- 
MANUEL SEEGER - CHRISTIAN FREVEL

Tabla 3: Características hidráulicas del perfil P1 (Leptic Haplogypsid), María de Huerva, campo abandonado hace unos 3 años en el momento del muestreo.

Se muestran la densidad aparente (DA), el volumen total de poros (PT),

los poros gruesos (PG), la CRAD, los parámetros de la

ecuación de van Genuchten $(a, n)$ y la conductividad hidráulica en

saturación (Kf).

Tabelle 3: Bodenbydraulische Kennwerte von Profil P1 (Leptic Haplogypsid), Maria de Huerva, zum Aufnabmezeitpunkt ca. 3 Jabre alte Brache. Es werden die Lagerungsdicbte $(D A)$, das Porenvolumen (PT), die Luftkapazität (PG), die effektiv nutzbare Feldkapazität (CRAD; kummuliert über die Bodensäule),

van-Genuchten Anpassungsparameter $(a, n)$ und die gesättigte Hydraulische Leitfäbigkeit (Kf) angegeben.

\begin{tabular}{|c|c|c|c|c|c|c|c|c|}
\hline Horizonte & $\begin{array}{c}\text { Limite } \\
{[\mathrm{cm}]}\end{array}$ & $\begin{array}{c}\mathrm{DA} \\
{\left[\mathrm{gcm}^{-3}\right]}\end{array}$ & $\begin{array}{c}\mathrm{PT} \\
{\left[\mathrm{cm}^{3} / \mathrm{cm}^{-3}\right]}\end{array}$ & $\begin{array}{c}\mathrm{PG} \\
{\left[\mathrm{cm}^{3} / \mathrm{cm}^{-3}\right]}\end{array}$ & $\begin{array}{c}\mathrm{CRAD} \\
{[\mathrm{mm}]}\end{array}$ & $\begin{array}{c}\mathrm{a} \\
{\left[\mathrm{hPa}^{-1}\right]}\end{array}$ & $\mathrm{n}$ & $\begin{array}{c}\mathrm{Kf} \\
{[\mathrm{mm} / \mathrm{h}]}\end{array}$ \\
\hline rAp1y & 15 & 1.37 & 0.45 & 0.11 & 29.7 & 0.0782 & 1.1594 & 38 \\
\hline rAp2y & 30 & 1.35 & 0.46 & 0.16 & 55.1 & 0.4899 & 1.1324 & 95 \\
\hline 2C & 60 & 1.50 & 0.44 & 0.03 & 137.6 & 0.0005 & 1.5762 & 7 \\
\hline
\end{tabular}

Tabla 4: Características hidráulicas del perfil P2 (Leptic Haplogypsid), María de Huerva, campo abandonado hace unos 65 años en el momento del muestreo.

Se muestran la densidad aparente (DA), el volumen total de poros (PT),

los poros gruesos (PG), la CRAD, los parámetros de la ecuación de van Genuchten $(\mathrm{a}, \mathrm{n})$ y la conductividad hidráulica en saturación (Kf).

Tabelle 4: Bodenbydraulische Kenmwerte von Profil P2 (Leptic Haplogypsid), Maria de

Huerva, zum Aufnabmezeitpunkt ca. 65 Jabre alte Brache. Es werden die

Lagerungsdichte (DA), das Porenvolumen (PT), die Luftkapazität (PG),

die effektiv nutzbare Feldkapazität (CRAD; kummuliert über die Bodensäule), van-Genuchten Anpassungsparameter $(a, n)$ und die gesättigte Hydraulische Leitfäbigkeit (Kf) angegeben.

\begin{tabular}{|l|c|c|c|c|c|c|c|c|}
\hline Horizonte & $\begin{array}{c}\text { Límite } \\
{[\mathrm{cm}]}\end{array}$ & $\begin{array}{c}\mathrm{DA} \\
{\left[\mathrm{gcm}^{-3}\right]}\end{array}$ & $\begin{array}{c}\mathrm{PT} \\
{\left[\mathrm{cm}^{3} / \mathrm{cm}^{-3}\right]}\end{array}$ & $\begin{array}{c}\mathrm{PG} \\
{\left[\mathrm{cm}^{3} / \mathrm{cm}^{-3}\right]}\end{array}$ & $\begin{array}{c}\text { CRAD } \\
{[\mathrm{mm}]}\end{array}$ & $\begin{array}{c}\mathrm{a} \\
{\left[\mathrm{hPa}^{-1}\right]}\end{array}$ & $\mathrm{n}$ & $\begin{array}{c}\mathrm{Kf} \\
{[\mathrm{mm} / \mathrm{h}]}\end{array}$ \\
\hline Apy2 & 28 & 1.35 & 0.43 & 0.15 & 44.0 & 0.3882 & 1.1690 & 189 \\
\hline By1 & 52 & 1.39 & 0.43 & 0.12 & 77.4 & 0.2777 & 1.1165 & 39 \\
\hline By2 & 76 & 1.29 & 0.45 & 0.11 & 131.7 & 0.0051 & 1.5286 & 22 \\
\hline
\end{tabular}




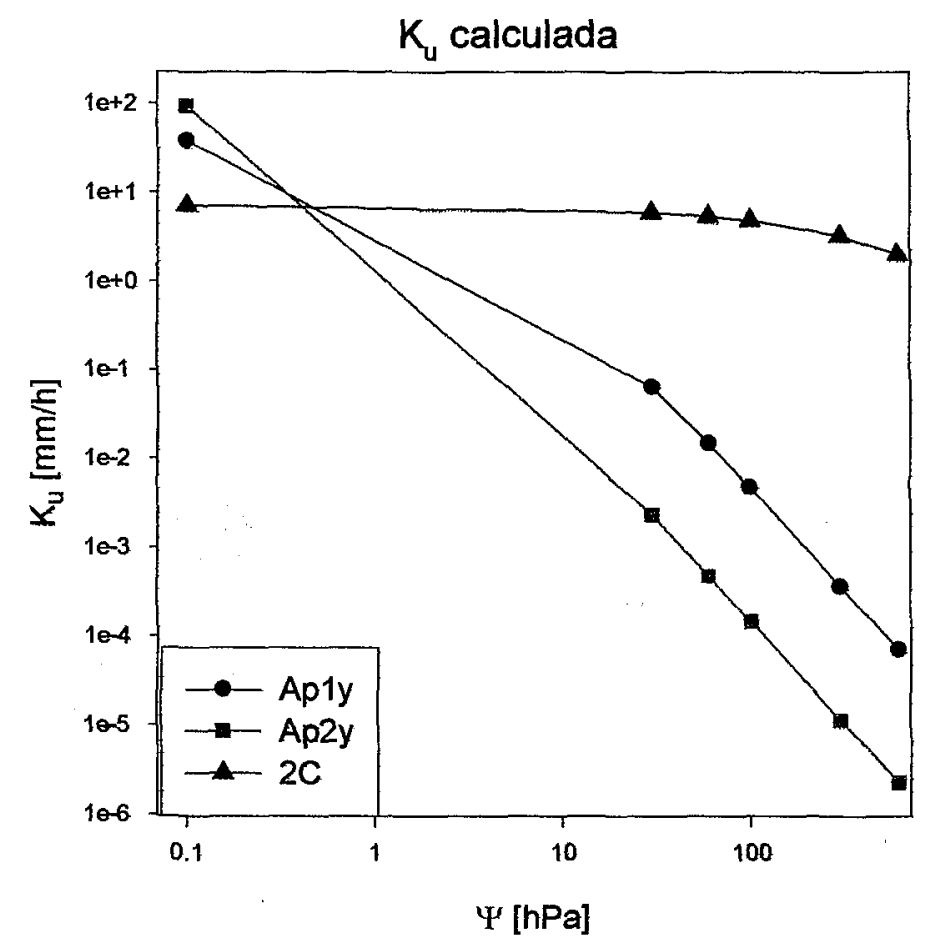

Fig. 2: Conductividad bidráulica del suelo no saturado $\left(K_{\mathcal{u}}\right)$ del perfil P1 (Leptic Haplogypsid) calculada a partir de la parametrización de la curva de valores de bumedad y pF. Tensión bidrica en el suelo $\psi$ en hPa. Se muestran los valores adquiridos de cada borizonte analizado, véase también Tabla 4. Se aprecia la similitud del comportamiento de los borizontes superficiales, al igual que la diferencia clara del borizonte subsuperficial.

Abb. 2: Ungesättigte bydraulische Leitfähigkeit $\left(K_{\mathcal{u}}\right)$ von Profil P1 (Leptic Haplogypsid), berechnet anhand der Parametrisierung der pF-Kurve nach van Genuchten. Tensionen $\psi$ in $\mathrm{bPa}$. Es sind die errechneten Werte jedes einzelnen Horizontes aufgetragen (siebe auch Tab. 4).

dad hasta un valor de $11 \%$. La CRAD acumulada hasta una profundidad de $76 \mathrm{~cm}$ es de $138 \mathrm{~mm}$. Por debajo del horizonte Ap la conductividad hidráulica disminuye sensiblemente, siendo sin embargo alta a lo largo de todo el perfil. Los horizontes Apy2 y By1 tienen un comportamiento hidráulico muy parecido con la disminución de la humedad del suelo, mientras que el horizonte subyacente mantiene valores relativamente altos hasta un $\mathrm{pF}=2$.

Los Leptic Haplogypsids del pie de ladera, que nunca fueron labrados, aparecen relativamente libres de perturbaciones. El epipedon conserva abundantes poros gruesos y una conductividad hidráulica alta (Tabla 5). A mayor profundidad, a causa del aumento de yesos y la disminución de la materia orgánica, disminuyen los poros medios, mientras la porosidad gruesa se mantiene, esencial- 
mente por la fuerte actividad de lombrices. Debajo de $60 \mathrm{~cm}$ de profundidad, estos últimos disminuyen también, hasta valores considerados no aptos para el crecimiento de raíces (Horn, 1992). Este fenómeno, al igual que la disminución de la conductividad hidráulica, se debe a la cementación del endopedion por la gran cantidad de yesos acumulados. Los valores de conductividad hidráulica disminuyen fuertemente en todo el perfil con el aumento de la tensión matricial, siendo la disminución en el epipedion considerablemente mayor.

Tabla 5: Caracteristicas hidráulicas del perfil P5 (Leptic Haplogypsid), María de Huerva, pie de ladera, presuntamente nunca labrado. Se muestran la densidad aparente (DA), el volumen total de poros (PT), los poros gruesos (PG), la CRAD, los parámetros de la ecuación de van Genuchten (a, n) y la conductividad hidráulica en saturación (Kf).

Tabelle 5: Bodenhydraulische Kennwerte von Profil P5 (Leptic Haplogypsid), Maria de Huerva, hangfußbereich, nie bearbeitet. Es werden die Lagerungsdichte (DA), das Porenvolumen (PT), die Luftkapazität (PG), die effektiv nutzbare Feldkapazität (CRAD; kummuliert über die Bodensäule), van-Genucbten Anpassungsparameter $(a, n)$ und die gesättigte Hydraulische Leitfähigkeit (Kf) angegeben.

\begin{tabular}{|l|c|c|c|c|c|c|c|c|}
\hline Horizonte & $\begin{array}{c}\text { Límite } \\
{[\mathrm{cm}]}\end{array}$ & $\begin{array}{c}\text { DA } \\
{\left[\mathrm{gcm}^{3}\right]}\end{array}$ & $\begin{array}{c}\mathrm{PT} \\
{\left[\mathrm{cm}^{3} / \mathrm{cm}^{-3}\right]}\end{array}$ & $\begin{array}{c}\mathrm{PG} \\
{\left[\mathrm{cm}^{3} / \mathrm{cm}^{-3}\right]}\end{array}$ & $\begin{array}{c}\text { CRAD } \\
{[\mathrm{mm}]}\end{array}$ & $\begin{array}{c}\mathrm{a} \\
{\left[\mathrm{hPa}^{-1]}\right]}\end{array}$ & $\mathrm{n}$ & $\begin{array}{c}\mathrm{Kf} \\
{[\mathrm{mm} / \mathrm{h}]}\end{array}$ \\
\hline Ah3 & 14 & 1.32 & 0.49 & 0.16 & 18.7 & 0.7656 & 1.1001 & 248 \\
\hline 2By & 26 & 1.35 & 0.42 & 0.16 & 29.6 & 35.0483 & 1.1216 & 101 \\
\hline 2C1 & 60 & 1.27 & 0.42 & 0.09 & 89.3 & 0.0776 & 1.1354 & 15 \\
\hline 2C2 & 80 & 1.29 & 0.41 & 0.05 & 131.1 & 0.0178 & 1.1715 & 7 \\
\hline
\end{tabular}

La profundidad de enraizamiento se ve limitada en toda la zona estudiada por contenidos en yesos muy elevados en profundidad, horizontes compactados o bancos de yesos aflorantes a escasa profundidad. Todos los suelos contienen por ello una CRAD baja (o media) en la zona enraizada. La conductividad hidráulica en saturación, en general alta a muy alta, disminuye drásticamente con la progresiva desecación de los suelos, especialmente en los epipedones estudiados. Este hecho, combinado con el encostramiento de todos los suelos de María de Huerva, provoca altos índices de escorrentía y bajas capacidades de infiltración. Las diferencias entre los suelos estudiados se deben primordialmente al tiempo transcurrido desde la ultima labor.

En situaciones topográficamente desfavorables, como las medias laderas y las cimas cercanas a las áreas de muestreo, se encuentran condiciones mas problemáticas para la retención de agua en el suelo. Contenidos en yesos extremos y 
FACTORES HÍDRICOS Y EROSIONABILIDAD EN EL GRADIENTE EDAFOCLIMÁTICO

mayor actividad geomorfodinámica limitan la profundidad edáfica, y ligado a ella, la reserva hídrica.

\subsection{Sabayés}

Los suelos característicos de la zona de Sabayés corresponden al subgrupo del Haplic Calcixerept. Áreas de menor espesor de suelo llevan a la clasificación de Ruptic-lithic Calcixerepts. A causa de acumulaciones hídricas subsuperficiales y superficiales se encuentran, en los bordes inferiores de los taludes de las terrazas, suelos de características hiodromorfas como los aeric Endoaquepts.

\subsubsection{Valores de erosionabilidad}

Los suelos de Sabayés son muy ricos en limos y arenas finas (entre $50 \%$ y $70 \%$ ) y pobres en materia orgánica, lo cual provoca una erosibilidad alta de la matriz de suelo, con valores $\mathrm{K}$ entre 0,39 y 0,32 . La acumulación residual de cantos en la superficie y los primeros $25 \mathrm{~cm}$ del perfil confirman este hecho, ya que demuestran que el suelo ha estado expuesto a una alta erosión. Esta acumulación, con coberturas entre un $10 \%$ y un $60 \%$ de la superficie, contribuye a reducir la erosionabilidad y aumenta la conductividad hidráulica en saturación. El factor $\mathrm{K}$ desciende así a valores medios y bajos.

\subsubsection{Parámetros hidráulicos}

La física de los suelos de Sabayés está claramente determinada por la fuerte acumulación de cantos en el epipedon. El espacio aprovechable como almacén hídrico es así muy limitado (Tabla 6 ). La CRAD a lo largo del perfil es baja, mientras que la conductividad hidráulica saturada es muy alta en los horizontes superficiales, disminuyendo fuertemente en los subyacentes. La evolución de la conductividad hidráulica no saturada es parecida en todos los horizontes, siendo la del horizonte Ck la más alta (Figura 3).

La zona cultivada con almendros se diferencia poco de la zona abandonada, aunque la labor constante impida la diferenciación de horizontes.

A pesar de que el comportamiento del suelo no saturado en Sabayés es muy parecido al determinado en María de Huerva, son de esperar comportamientos muy diferentes, ya que la riqueza en piedras estabiliza los sistemas de poros gruesos y medios en sus bordes, a la vez que una cubierta pedregosa los protege del encostramiento. La infiltración será así notablemente mayor.

\subsection{Bentué de Rasal}

El área de Bentué de Rasal muestra una variedad amplia de suelos de los grupos ústicos y xéricos de las ordenes de los Alfisoles, los Entisoles y los 
Inceptisoles. En las terrazas de menor pendiente se encuentran dos variedades de Inceptic Haplustalfs: una en la zonas con un cono aluvial de considerable espesor, y una variante calcareous, con alto contenido en carbonato cálcico, en las zonas donde la labor ha mezclado los materiales aluviales con las margas subyacentes. En los bordes inferiores de los taludes se encuentran Lithic Xerorthents, y fuera de los campos abandonados, donde sedimentan los materiales transportados, se encuentran Typic Ustorthents. Las zonas de contacto entre estos últimos suelos esta formada por Calcic Haplustepts.

Los campos abandonados de mayor edad, expuestos al nordeste, estan ocupados por Typic y Lithic Ustorthents.

Tabla 6: Características hidráulicas del perfil P6 (Haplic Calcixerept), Sabayés, solana, antiguos viñedos abancalados, despues de su abandono fueron quemados regularmente para pasto. Se muestran la densidad aparente (DA), el volumen total de poros (PT), los poros gruesos (PG), la CRAD, los parámetros de la ecuación de van Genuchten $(a, n)$ y la conductividad hidráulica en saturación (Kf).

Tabelle 6: Bodenbydraulische Kennwerte von Profil P6 (Haplic Calcixerept), Sabayés, südexponierter Hang, ebemalige Weinbauterrassen, nach aufgabe regelmäßig gebrannt zur Weideverbesserung. Es werden die Lagerungsdichte (DA), das Porenvolumen (PT), die

Luftkapazität (PG), die effektiv nutzbare Feldkapazität (CRAD; kummuliert über die

Bodensäule), van-Genuchten Anpassungsparameter $(a, n)$ und die gesättigte Hydraulische Leitfäbigkeit (Kf) angegeben.

\begin{tabular}{|c|c|c|c|c|c|c|c|c|}
\hline Horizonte & $\begin{array}{c}\text { Límite } \\
{[\mathrm{cm}]}\end{array}$ & $\begin{array}{c}\mathrm{DA} \\
{\left[\mathrm{gcm}^{-3}\right]}\end{array}$ & $\begin{array}{c}\mathrm{PT} \\
{\left[\mathrm{cm}^{3} / \mathrm{cm}^{-3}\right]}\end{array}$ & $\begin{array}{c}\mathrm{PG} \\
{\left[\mathrm{cm}^{3} / \mathrm{cm}^{-3}\right]}\end{array}$ & $\begin{array}{c}\mathrm{CRAD} \\
{[\mathrm{mm}]}\end{array}$ & $\begin{array}{c}\mathrm{a} \\
{\left[\mathrm{hPa}^{-1}\right]}\end{array}$ & $\mathrm{n}$ & $\begin{array}{c}\mathrm{Kf} \\
{[\mathrm{mm} / \mathrm{h}]}\end{array}$ \\
\hline $\mathrm{Ap}$ & 25 & 1.41 & 0.36 & 0.12 & 30.4 & 0.4854 & 1.1148 & 358 \\
\hline $\mathrm{Bk}$ & 50 & 1.50 & 0.41 & 0.15 & 70.8 & 0.3191 & 1.1314 & 343 \\
\hline $\mathrm{Ck}$ & 75 & 1.59 & 0.37 & 0.08 & 118.3 & 0.0222 & 1.1673 & 15 \\
\hline
\end{tabular}

\subsubsection{Valores de erosionabilidad}

La erosionabilidad de los horizontes superficiales de Bentué de Rasal es alta a muy alta (valores de $\mathrm{K}$ entre 0,32 y 0,75 ). Sólamente el Haplustalf y el typic Ustochrept, con una conductividad hidráulica extremadamente alta, presentan una erosibilidad media en superficie. Al igual que en Sabayés, la acumulacion de cantos en superficie, con una cobertura de hasta el $90 \%$, hacen disminuir el factor $\mathrm{K}$ a valores muy bajos y bajos. 


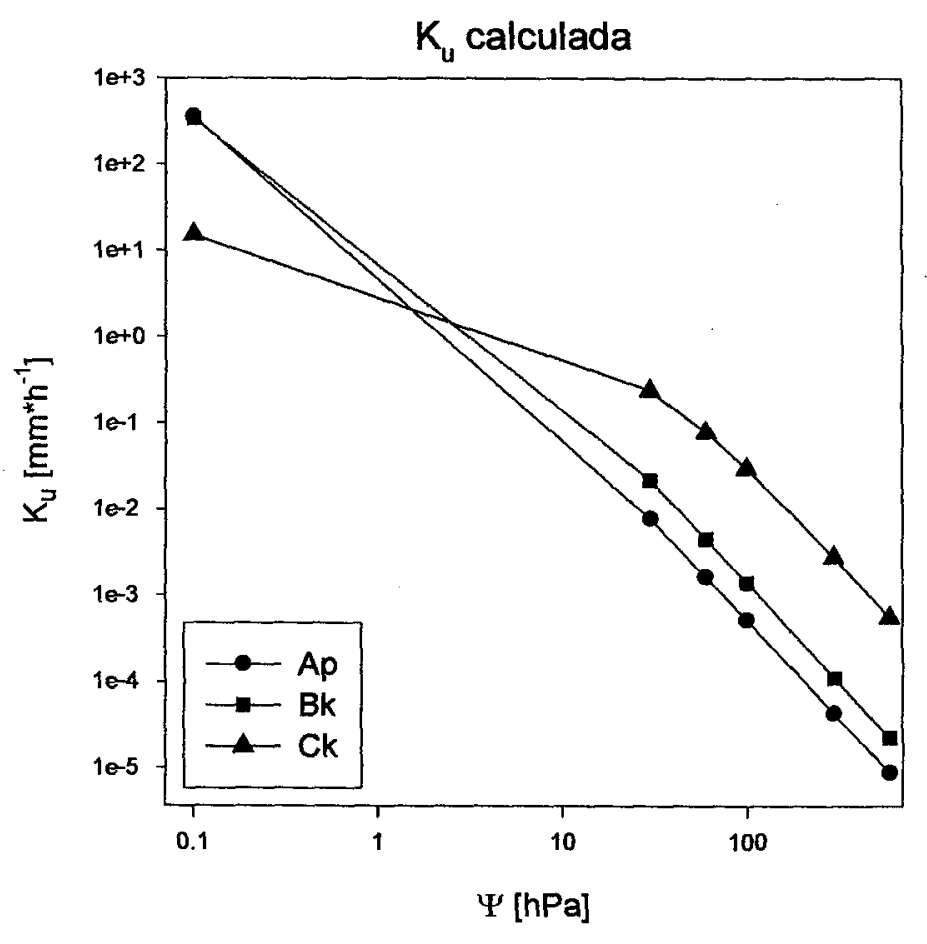

Figura 3: Conductividad bidráulica del suelo no saturado $\left(K_{\mathcal{u}}\right)$ del perfil P6 (Haplic Calcixerept) calculada a partir de la parametrización de la curva de valores de bumedad y pF. Tensión bidrica en el suelo $\psi$ en bPa. Se muestran los valores adquiridos de cada borizonte analizado, véase también Tabla 7.

Se aprecia la similitud del comportamiento de los borizontes superficiales, al igual que la diferencia clara del borizonte subsuperficial, que reflejan asi la determinante estructural del laboreo.

Abb. 3: Ungesättigte bydraulische Leitfäbigkeit $\left(K_{\mathcal{W}}\right)$ von Profil P6 (Haplic Calcixerept), berechnet anhand der Parametrisierung der pF-Kurve nach van Genuchten. Tensionen $\psi$ in $\mathrm{bPa}$. Es sind die errechneten Werte jedes einzelnen Horizontes aufgetragen (siebe auch Tab. 7).

\subsubsection{Parámetros hidráulicos}

A causa de la variabilidad de los suelos de Bentué de Rasal es difícil reconocer distribuciones espaciales de sus características. Incluso en una misma terraza pueden hallarse suelos de características muy diferentes, independientemente del uso anterior y la antigüedad de su abandono. A modo de ejemplo se presentarán dos suelos:

El Lithic Xerorthent (Perfil 8, Tabla 7) se localiza en una zona de escaso espesor de suelo, cuya porosidad disminuye a la mitad a causa del incremento de cantos en el Horizonte Ah/Cv. La capacidad de almacenamiento hídrico es muy esca- 
MANUEL SEEGER - CHRISTIAN FREVEL

sa, mientras que la conductividad hidráulica en saturación es muy alta en el epipeon y disminuye en el endopedon. La evolución del comportamiento hidráulico del suelo no saturado es parecida en los dos horizontes: por encima de un pF de 2,0 la conductividad es muy baja.

Tabla 7: Características hidráulicas del perfil P8 (Lithic Xerorthent), Bentué de Rasal, campo abandonado hace 30 años, ocasionalmente pastoreado.

Se muestran la densidad aparente (DA), el volumen total de poros (PT),

los poros gruesos (PG), la CRAD, los parámetros de la

ecuación de van Genuchten $(a, n)$ y la conductividad hidráulica en saturación (Kf).

Tabelle 7: Bodenbydraulische Kennwerte von Profil P8 (Litbic Xerorthent), Bentué de

Rasal, seit etwa 30 Jabren brachliegender Acker, gelegentlich beweidet.

Es werden die Lagerungsdichte (DA), das Porenvolumen (PT), die Luftkapazität (PG), die effektiv nutzbare Feldkapazität (CRAD; kummuliert über die Bodensäule), van-Genuchten Anpassungsparameter $(a, n)$ und die gesättigte Hydraulische Leitfäbigkeit (Kf) angegeben.

\begin{tabular}{|c|c|c|c|c|c|c|c|c|}
\hline Horizonte & $\begin{array}{c}\text { Límite } \\
{[\mathrm{cm}]}\end{array}$ & $\begin{array}{c}\mathrm{DA} \\
{\left[\mathrm{gcm}^{-3}\right]}\end{array}$ & $\begin{array}{c}\mathrm{PT} \\
{\left[\mathrm{cm}^{3} / \mathrm{cm}^{-3}\right]}\end{array}$ & $\begin{array}{c}\text { PG } \\
{\left[\mathrm{cm}^{3} / \mathrm{cm}^{-3}\right]}\end{array}$ & $\begin{array}{c}\mathrm{CRAD} \\
{[\mathrm{mm}]}\end{array}$ & $\begin{array}{c}\mathrm{a} \\
{\left[\mathrm{hPa}^{-1]}\right]}\end{array}$ & $\mathrm{n}$ & $\begin{array}{c}\mathrm{Kf} \\
{[\mathrm{mm} / \mathrm{h}]}\end{array}$ \\
\hline $\mathrm{Ah}$ & 12 & 1.36 & 0.42 & 0.19 & 17.4 & 1.0511 & 1.1503 & 388 \\
\hline $\mathrm{Ah} / \mathrm{Cv}$ & 28 & 1.73 & 0.24 & 0.08 & 32.1 & 0.2421 & 1.1311 & 66 \\
\hline
\end{tabular}

El perfil P9 es, en cambio, un suelo profundo y de varias capas, con abundantes raíces hasta gran profundidad. La acumulación de arcillas en el endopedon provoca un fuerte descenso de la conductividad hidráulica hasta valores medios (Tabla 8). Existen abundantes poros gruesos en los primeros $40 \mathrm{~cm}$, disminuyendo su cantidad hasta un valor medio. La CRAD acumulada hasta una profundidad de $65 \mathrm{~cm}$ es baja, aunque las raíces llegan a alcanzar profundidades mayores, con lo cual se puede considerar una CRAD media. En todos los horizontes se puede observar una disminución doble logarítmica de la conductividad hidráulica en el suelo no saturado, encontrándose los valores mas bajos en los horizontes Ah1 y Bt (Figura 4).

Se puede resumir, que en el área de Bentué de Rasal, los epipedones muestran altos valores de conductividad hidráulica. Esta disminuye fuertemente con la desecación del suelo. El alto contenido en macroporos, protegidos por la cobertura pedregosa como en Sabayés, permite de todas formas una rápida infiltración del agua. Se observa, sin embargo, esencialmente en los suelos más someros, que el agua puede infiltrar fácilmente hasta el sistema de poros y brechas de las mar- 
FACTORES HÍDRICOS Y EROSIONABILIDAD EN EL GRADIENTE EDAFOCLIMÁTICO

Tabla 8: Características hidráulicas del perfil P9 (Inceptic Haplustalf), Bentué de Rasal, campo abandonado hace unos 30 años, ocasionalmente pastoreado. Se muestran la densidad aparente (DA), el volumen total de poros (PT), los poros gruesos (PG),

la CRAD, los parámetros de la ecuación de van Genuchten $(a, n)$ y la conductividad hidráulica en saturación (Kf).

Tabelle 8: Bodenbydraulische Kennwerte von Profil P9 (Inceptic Haplustalf), Bentué de Rasal, seit etwa 30 Jabren brachliegender Acker, gelegentlich beweidet. Es werden die Lagerungsdichte (DA), das Porenvolumen (PT), die Luftkapazität (PG), die effektiv nutzbare Feldkapazität (CRAD; kummuliert über die Bodensäule), van-Genuchten Anpassungsparameter $(a, n)$ und die gesättigte Hydraulische Leitfäbigkeit (Kf) angegeben.

\begin{tabular}{|c|c|c|c|c|c|c|c|c|}
\hline Horizonte & $\begin{array}{c}\text { Límite } \\
{\left[\mathrm{cm}^{2}\right]}\end{array}$ & $\begin{array}{c}\mathrm{DA} \\
{\left[\mathrm{gcm}^{-3}\right]}\end{array}$ & $\begin{array}{c}\text { PT } \\
{\left[\mathrm{cm}^{3} / \mathrm{cm}^{-3}\right]}\end{array}$ & $\begin{array}{c}\mathrm{PG} \\
{\left[\mathrm{cm}^{3} / \mathrm{cm}^{-3}\right]}\end{array}$ & $\begin{array}{c}\text { CRAD } \\
{[\mathrm{mm}]}\end{array}$ & $\begin{array}{c}\mathrm{a} \\
{\left[\mathrm{hPa}^{-1}\right]}\end{array}$ & $\mathrm{n}$ & $\begin{array}{c}\mathrm{Kf} \\
{[\mathrm{mm} / \mathrm{h}]}\end{array}$ \\
\hline $\mathrm{Ah} 1$ & 8 & 1.16 & 0.54 & 0.24 & 15.2 & 1.1027 & 1.1425 & 249 \\
\hline $\mathrm{Ah} 2$ & 25 & 1.49 & 0.42 & 0.12 & 45.4 & 1.1141 & 1.1392 & 310 \\
\hline $\mathrm{Bt}$ & 40 & 1.47 & 0.43 & 0.03 & 69.4 & 0.2253 & 1.1109 & 23 \\
\hline $2 \mathrm{Cv}$ & 65 & 1.69 & 0.36 & 0.09 & 111.2 & 0.0505 & 1.1529 & 73 \\
\hline
\end{tabular}

gas subyacentes, de modo que el agua desaparece en el subsuelo sin penetrar en la matriz. Así, la baja capacidad de retención hídrica del suelo se ve desfavorecida además por una difícil recarga hídrica.

\subsection{Arnás}

Los suelos de Arnás, en plena transición hacia el Pirineo central húmedo, pertenecen a los grupos de los Entisoles, los Inceptisoles, los Mollisoles y los Alfisoles.

En los bordes externos de los antiguos campos aterrazados, y en los límites con las laderas convexas no aterrazadas se han identificado Lithic y Typic Haplustepts. Las terrazas más amplias, creadas en un derrubio de ladera, muestran en su parte inferior, de poca inclinación y relativamente pobre en piedras, suelos del tipo Oxyaquic Vertic Haplustalf, de considerable profundidad. Los suelos de la zona superior de esta misma terraza, con mayor inclinación y marcados por procesos erosivos, pertenecen al subgrupo de los Aquic Calciustept.

En la cima de la ladera se encuentran, además, suelos del tipo Lithic Rendoll.

\subsubsection{Valores de erosionabilidad}

La matriz de los suelos de Arnás es, a causa del alto contenido en limos, y a pesar de su alto contenido en materia orgánica, altamente susceptible a la erosión 


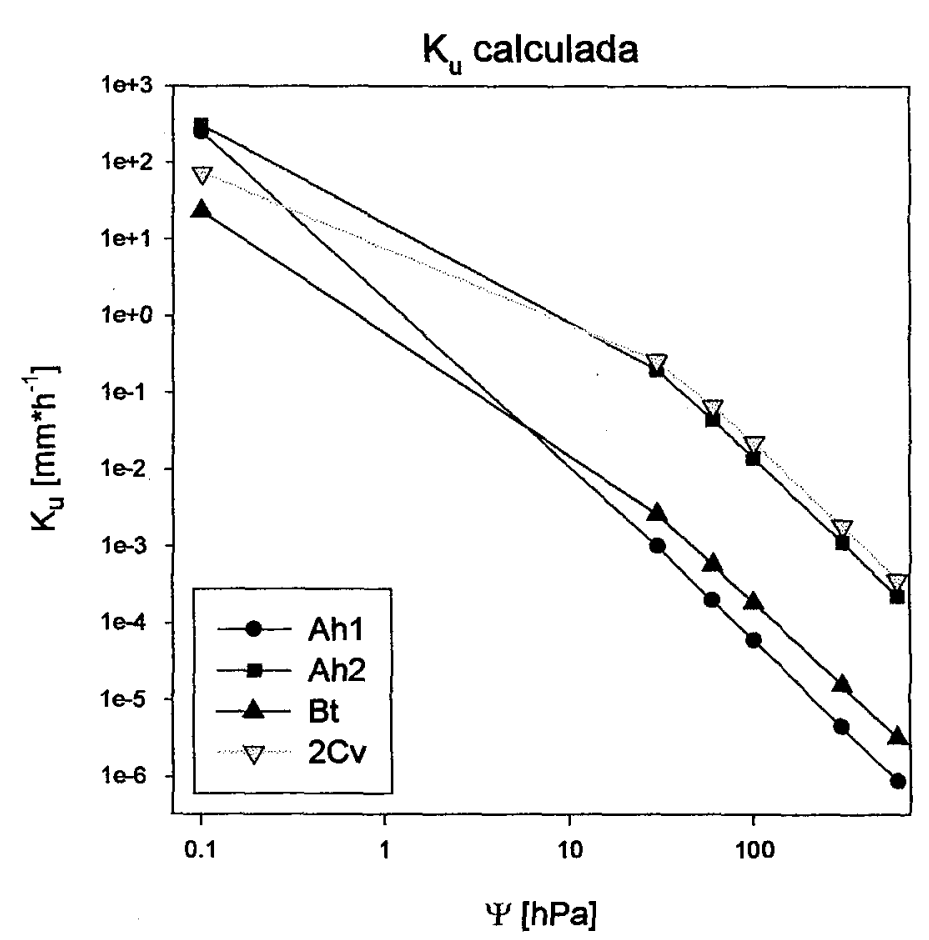

Figura 4: Conductividad bidrâulica calculada para condiciones no saturadas $\left(K_{\mathcal{u}}\right)$ del perfil P9 (Inceptic Haplustalf) calculada a partir de la parametrización de la curva de valores de bumedad y pF. Tensión bidriça en el suelo $\psi$ en bPa. Se muestran los valores adquiridos de cada borizonte analizado, véase también Tabla 9. Abb. 4: Ungesättigte bydraulische Leitfähigkeit $\left(K_{\mathcal{W}}\right)$ von Profil P9 (Inceptic Haplustalf), berechnet anhand der Parametrisierung der pF-Kurve nach van Genucbten. Tensionen $\psi$ in bPa. Es sind die errechneten Werte jedes einzelnen Horizontes aufgetragen (siebe auch Tab. 9).

hídrica. Los suelos de la terraza inferior muestran valores del factor $\mathrm{K}$ entre 0,31 y 0,37 . Los suelos de las laderas, más ricos en arenas en los horizontes superficiales, sólo alcanzan valores entre 0,23 y 0,25 . Este incremento de la fracción de arena en los horizontes superficiales es, a su vez, un indicio de la erosión sufrida por estos suelos. El lithic Rendoll del collado muestra, en cambio, por las abundantes arcillas y materia orgánica, valores bajos de erosionabilidad.

En Arnás tambien se observa una acumulación de piedras importante en la superficie, con lo cual el potencial de erosión se reduce considerablemente, como en el caso de las laderas, que muestran erosionabilidades muy bajas.

\subsubsection{Parámetros hidráulicos}

Los suelos de Arnás muestran un alto contenido en arcillas, lo cual los diferencia de los suelos presentados en apartados anteriores. La elevada cantidad de 
piedras solamente permitió disponer de muestras no perturbadas del perfil P13, un Oxyaquic Vertic Haplustalf. De todas formas, en el campo puede observarse una fuerte variación temporal de los macroporos ligada a fenómenos de expansión y retracción de las arcillas. Así, los valores calculados han de ser vistos, más que en otras áreas muestreadas, como valores orientativos (Tabla 9).

Tabla 9: Características hidráulicas del perfil P13 (Oxyaquic Vertic Haplustalf), Arnás, campo abandonado hace aprox. 30 años, intensamente pastoreado.

Se muestran la densidad aparente (DA), el volumen total de poros (PT),

los poros gruesos (PG), la CRAD, los parámetros de la ecuación

de van Genuchten $(\mathrm{a}, \mathrm{n})$ y la conductividad hidráulica en saturación (Kf).

Tabelle 9: Bodenhydraulische Kennwerte von Profil P13 (Oxyaquic Vertic Haplustalf),

Arnâs, seit etwa 30 Jabren brachliegender Acker, intensiv beweidet. Es werden die

Lagerungsdichte (DA), das Porenvolumen (PT), die Luftkapazität (PG), die effektiv nutzbare Feldkapazität (CRAD; kummuliert über die Bodensäule), van-Genuchten

Anpassungsparameter $(a, n)$ und die gesättigte Hydraulische Leitfäbigkeit

(Kf) angegeben.

\begin{tabular}{|c|c|c|c|c|c|c|c|c|}
\hline Horizonte & $\begin{array}{c}\text { Limite } \\
{[\mathrm{cm}]}\end{array}$ & $\begin{array}{c}\mathrm{DA} \\
{\left[\mathrm{gcm}^{-3}\right]}\end{array}$ & $\begin{array}{c}\mathrm{PT} \\
{\left[\mathrm{cm}^{3} / \mathrm{cm}^{-3}\right]}\end{array}$ & $\begin{array}{c}\mathrm{PG} \\
{\left[\mathrm{cm}^{3} / \mathrm{cm}^{-3}\right]}\end{array}$ & $\begin{array}{c}\mathrm{CRAD} \\
{[\mathrm{mm}]}\end{array}$ & $\begin{array}{c}\mathrm{a} \\
{\left[\mathrm{hPa}^{-1}\right]}\end{array}$ & $\mathrm{n}$ & $\begin{array}{c}\mathrm{Kf} \\
{[\mathrm{mm} / \mathrm{h}]}\end{array}$ \\
\hline $\mathrm{Ap} 2$ & 17 & 1.30 & 0.38 & 0.10 & 59.4 & 0.0480 & 1.16 & 1156 \\
\hline $\mathrm{Bt}$ & 35 & 1.60 & 0.35 & 0.07 & 85.9 & 0.3191 & 1.13 & 10 \\
\hline $2 \mathrm{Btg}$ & 65 & 1.70 & 0.36 & 0.01 & 113.7 & $1.33 \mathrm{e}-3$ & 1.26 & 6 \\
\hline
\end{tabular}

La conductividad hidráulica del epipedon es muy alta, disminuyendo en profundidad. El contenido en poros gruesos disminuye desde valores medios en el horizonte superficial hasta un valor muy bajo en el horizonte 2Btg, debajo de $35 \mathrm{~cm}$ de profundidad. La CRAD es baja hasta una profundidad de $65 \mathrm{~cm}$. Por debajo no se observan raíces, debido a las características hidromorfas del endopedon. Esto sugiere que puede haber una constante recarga hídrica desde el subsuelo, aunque la conductividad hidráulica del horizonte Bt no saturado es extremadamente baja (Figura 5).

El comportamiento hidráulico de los suelos de Arnás es muy complejo. Los suelos secos tienen una baja conductividad hidráulica de la matriz, aunque pueden mostrar grietas de retracción de las arcillas, que permiten una infiltración rápida y hasta grandes profundidades. Si éstas han sido selladas por material erosionado o por el pisoteo del ganado, las precipitaciones pueden generar rápidamente escorrentía superficial. Lo mismo se observa con los suelos muy húmedos, ya que en este caso las grietas desaparecen. 


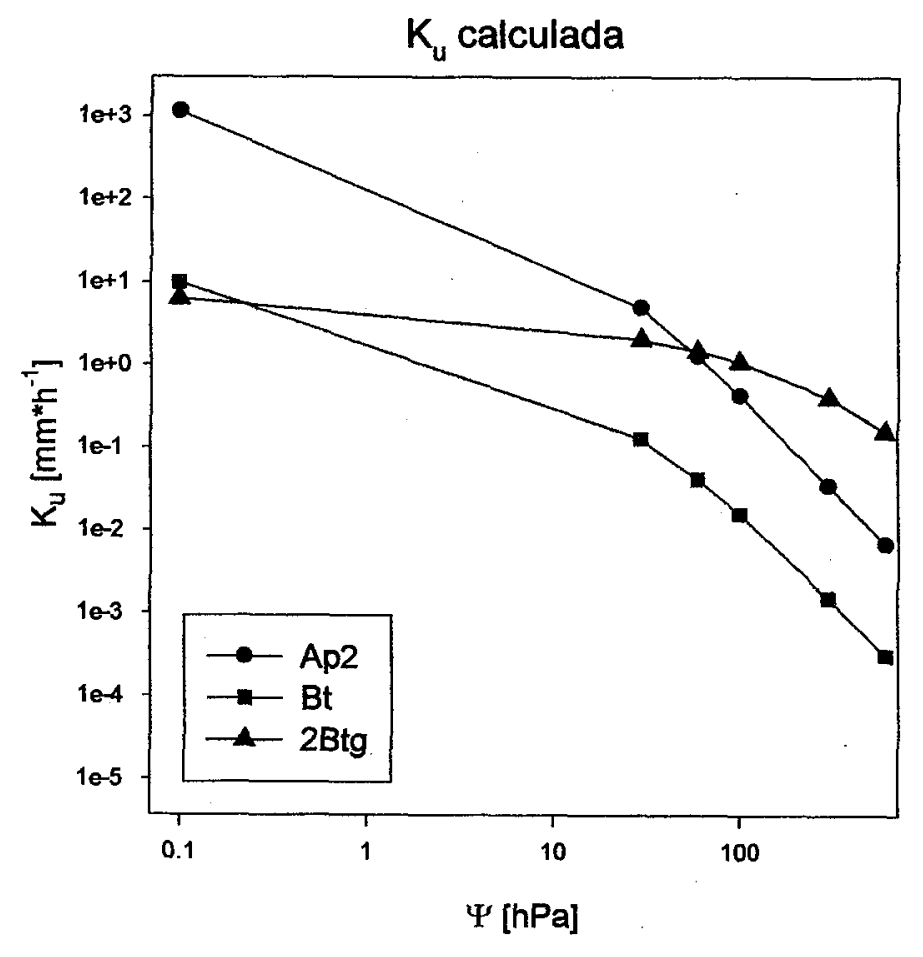

Figura 5: Conductividad bidráulica calculada para condiciones no saturadas $\left(K_{u}\right)$ del perfil P13 (Oxyaquic Vertic Haplustalf) calculada a partir de la parametrización de la curva de valores de bumedad y pF. Tensión bídrica en el suelo $\psi$ en hPa. Información detallada de los valores hidraúlicos del perfil en Tabla 10.

Se reconoce fácilmente la evolución no bomogénea de la conductividad con tensiones bidráulicas crecientes.

Abb. 5: Ungesättigte bydraulische Leitfäbigkeit $\left(K_{\mathcal{u}}\right)$ von Profil P13 (Oxyaquic Vertic Haplustalf), berechnet anband der Parametrisierung der $p F-K u r v e ~ n a c h$ van Genuchten. Tensionen $\psi$ in bPa. Es sind die errechneten Werte jedes einzelnen Horizontes aufgetragen (siebe auch Tab. 9).

\subsection{Aisa}

En la zona del Cubilar de Rigüelo, en el área de estudio de Aísa, se encuentran suelos de los grupos cryicos de los ordenes de los Alfisoles y los Entisoles. El Lithic Cryorthent de la ladera media superior es un suelo poco evolucionadocon abundantes cantos de caliza. Esta capa forma, además, los horizontes superficiales del Aquic Cryoboralf, superponiéndose a un horizonte formado a partir del flysch, fuertemente meteorizado. En pequeños rellanos bien drenados, y en áreas no deslizadas se ha podido formar un Mollic Dystrocryept ácido de considerable espesor. 


\subsubsection{Valores de erosionabilidad}

La fuerte erosividad de las precipitaciones y la energía del relieve en alta montaña se ven reflejados en los suelos compuestos por varias capas de diferente origen. La erosionabilidad de los suelos, ricos en limos y arenas finas, se ve ligeramente moderada por el alto contenido en materia orgánica hasta valores altos del factor $\mathrm{K}$, de aproximadamente 0,38 .

El Cryorthent esta protegido del efecto de impacto de las gotas de la lluvia por la pedregosidad superficial, con la cual la erosionabilidad se reduce a un valor bajo. A causa del deslizamiento de capas enteras del suelo, aflora en algunas áreas el endopedon sujeto a elevada erosionabilidad.

\subsubsection{Parámetros hidráulicos}

El Mollic Dystrocryept, formado sobre substratos poco perturbados, contiene abundante materia orgánica, y el horizonte superficial aparece intensamente enraizado. Se han estimado densidades aparentes bajas y una porosidad total muy elevada (Tabla 10). En cambio, la CRAD total del suelo es baja. La conductividad hidráulica es media hasta $60 \mathrm{~cm}$ de profundidad y por debajo solamente se obtiene una conductividad baja. En el suelo no saturado la conductividad hidráulica disminuye fuertemente, manteniéndose los niveles más altos en el horizonte Ah (Figura 6).

Tabla 10: Características hidráulicas del perfil P14 (Mollic Dystricryept), Aísa, pasto de verano para ganado bovino. Se muestran la densidad aparente (DA), el volumen total

de poros (PT), los poros gruesos (PG), la CRAD, los parámetros de la ecuación de van Genuchten $(\mathrm{a}, \mathrm{n})$ y la conductividad hidráulica en saturación (Kf).

Tabelle 10: Bodenbydraulische Kennwerte von Profil P14 (Mollic Dystrocryept), Aisa,

Somerbochweide. Es werden die Lagerungsdichte (DA), das Porenvolumen (PT), die Luftkapazität (PG), die effektiv nutzbare Feldkapazität (CRAD;

kummuliert über die Bodensäule), van-Genucbten Anpassungsparameter $(a, n)$ und die gesättigte Hydraulische Leitfähigkeit (Kf) angegeben.

\begin{tabular}{|c|c|c|c|c|c|c|c|c|}
\hline Horizonte & $\begin{array}{c}\text { Límite } \\
{[\mathrm{cm}]}\end{array}$ & $\begin{array}{c}\text { DA } \\
{\left[\mathrm{gcm}^{-3}\right]}\end{array}$ & $\begin{array}{c}\mathrm{PT} \\
{\left[\mathrm{cm}^{3} / \mathrm{cm}^{-3}\right]}\end{array}$ & $\begin{array}{c}\text { PG } \\
{\left[\mathrm{cm}^{3} / \mathrm{cm}^{-3}\right]}\end{array}$ & $\begin{array}{c}\text { CRAD } \\
{[\mathrm{mm}]}\end{array}$ & $\begin{array}{c}\mathrm{a} \\
{\left[\mathrm{hPa}^{-1}\right]}\end{array}$ & $\mathrm{n}$ & $\begin{array}{c}\mathrm{Kf} \\
{[\mathrm{mm} / \mathrm{h}]}\end{array}$ \\
\hline $\mathrm{Ah}$ & 18 & 0.88 & 0.66 & 0.20 & 47.8 & 0.1723 & 1.1291 & 192 \\
\hline $2 \mathrm{AB}$ & 28 & 1.06 & 0.59 & 0.20 & 63.9 & 4.0097 & 1.1000 & 248 \\
\hline $2 \mathrm{Bw}$ & 60 & 1.14 & 0.57 & 0.20 & 84.8 & 2.3479 & 1.1000 & 130 \\
\hline $3 \mathrm{BC}$ & 95 & 1.17 & 0.53 & 0.15 & 111.4 & n. B. & n. B. & 29 \\
\hline
\end{tabular}




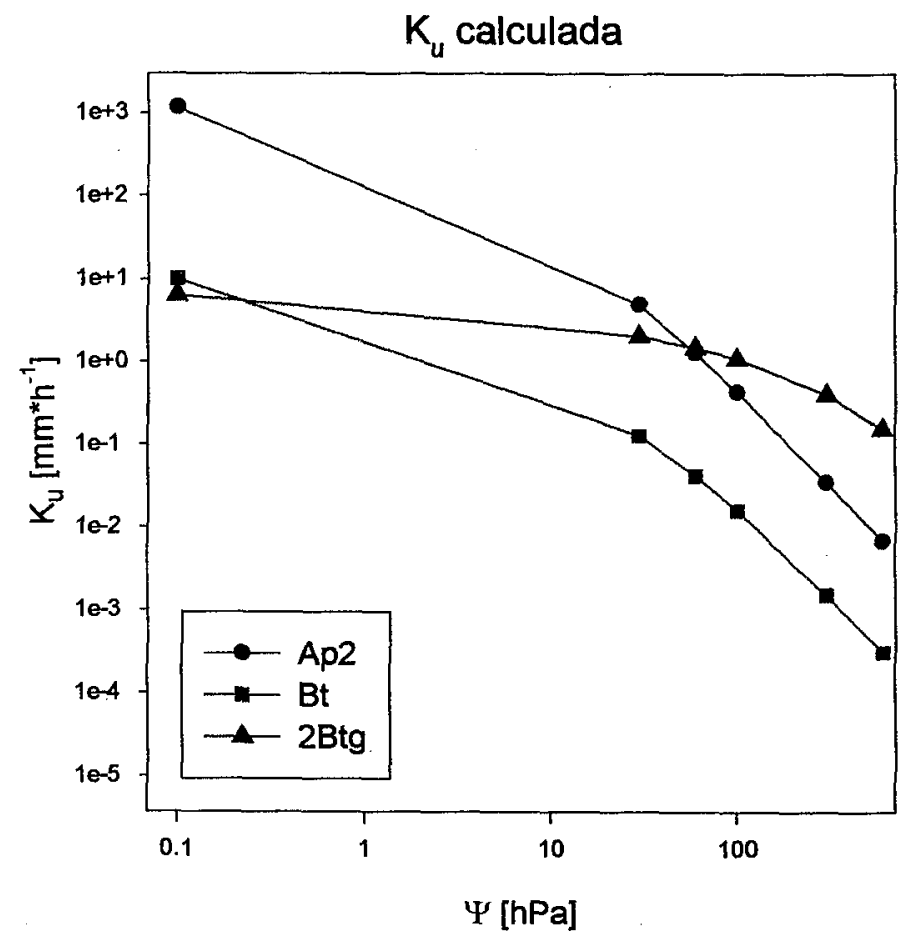

Figura 6: Conductividad bidráulica calculada para condiciones no saturadas $\left(K_{u}\right)$ del perfil P14 (Mollic Dystrocryept) calculada a partir de la parametrización de la curva de valores de bumedad y pF. Tensión hidrica en el suelo $\psi$ en bPa. Se muestran los valores adquiridos de cada borizonte analizado, véase también Tabla 11.

Abb. 6: Ungesättigte bydraulische Leitfäbigkeit $\left(K_{u}\right)$ von Profil P14 (Mollic Dystrocryept), berechnet anhand der Parametrisierung der pF-Kurve nach van Genuchten. Tensionen $\psi$ in bPa. Es sind die errechneten Werte jedes einzelnen Horizontes aufgetragen (siebe auch Tab. 9).

El Aquic Cryoboralf, formado en la zona deslizada, muestra, en cambio, porosidades y cantidades de poros gruesos decrecientes con la profundidad. La conductividad hidráulica disminuye a su vez de valores muy altos a valores medios. La evolución de los valores de Ku son similares en todos los horizontes, manteniéndose los valores más altos en el horizonte BtC.

\section{Discusiōn y conclusiones}

Se han estudiado los factores endógenos que favorecen la erosión del suelo y las características hidráulicas de las diferentes unidades caracterizadas en las áreas de estudio.

Los suelos de María de Huerva muestran, a causa del alto contenido en limos y el bajo contenido de materia orgánica, valores de erosionabilidad alta a muy alta. 
Esta se ve favorecida, además, por la formación de costras edáficas, que disminuyen considerablemente la capacidad de infiltración del suelo. En este sentido son los suelos del tipo Typic Haplogypsid los más favorables de la zona. En cambio, los suelos de Arnás, Bentué de Rasal y Sabayés, con importantes acumulaciones residuales de piedras en superficie como resultado de la erosión, o acumulación de materia orgánica, muestran valores del factor $\mathrm{K}$ considerablemente menores. Así, es evidente que entre la Depresión Central del Ebro y las Sierras Interiores del Pirineo existe una clara tendencia a la disminución de la erosionabilidad (véase Figura 7, izq.). Este fenómeno esta, sin embargo, estrechamente ligado a la pedregosidad de los suelos, ya que descartando la moderación de la erosionabilidad por parte de las piedras (Figura 7 , dcha.), se reconoce que la matriz de todos los suelos estudiados es entre medianamente y altamente susceptible a la erosión hídrica. Este hecho es muy importante para los suelos de Bentué de Rasal y Arnás, ya que aquí se pueden producir fenómenos de crioturbación, que transportan grandes cantidades de material fino a la superficie de donde puede ser fácilmente erosionado. Young et al. (1990) confirman que los suelos adquieren los valores más altos del factor $\mathrm{K}$ con la superficie recién descongelada.

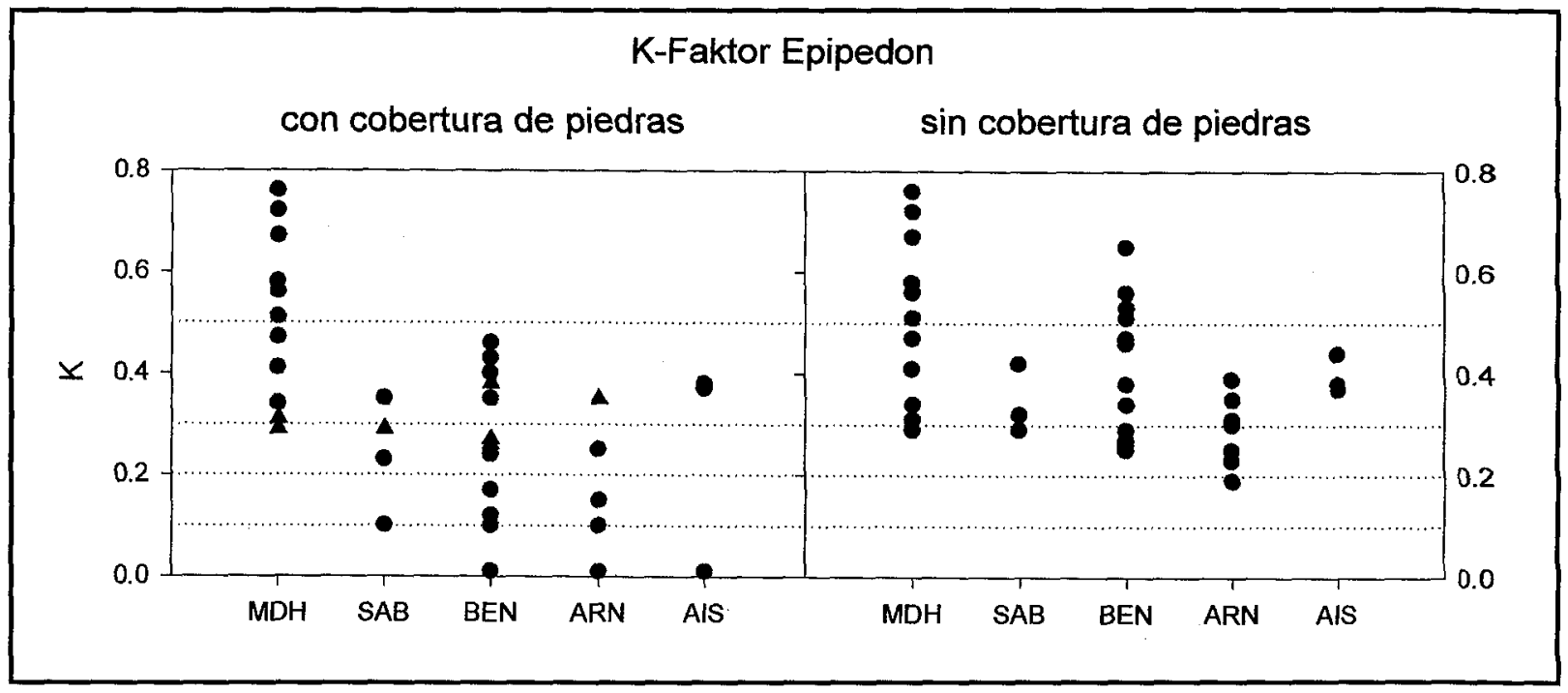

Figura 7: Valores del factor $K$ de los suelos analizados - a la izquierda incorporando en los cálculos la pedregosidad, a la derecha sin considerarla.

Además, se ban incorporado (triángulos), los valores $K$ de las heces de lombriz analizadas. Se observa la disminucion de la erosionabilidad de los suelos a causa de la pedregosidad de estos, exceptuando los suelos de María de Huerva, que carece de material grueso.

Abb. 7: K-Faktoren der untersucbten Böden - links unter Berücksicbtigung des Skelettgehaltes, rechts obne.

Zusätzlich sind die Werte der K-Faktoren für Wurmlosung (dreiecke) aufgenommen. 
En María de Huerva la erosionabilidad de los suelos se ve disminuida por la actividad de las lombrices, que transportan abundante material pobre en yesos a la superficie y lo enriquecen con materia orgánica, estabilizando así la estructura. En cambio, en Arnás, la actividad de los animales del suelo tiene una influencia negativa sobre la erosionabilidad del suelo, por su selección granulométrica de materiales limosos y arenosos y su transporte a la superficie. Los resultados sugieren que la actividad de la fauna es un factor estabilizador del suelo en la Depresión del Ebro, mientras que en las cotas más altas, esencialmente Arnás y Aísa, el material transportado por esa actividad a la superficie constituye una gran fuente de sedimentos.

Los parámetros hidráulicos, como la porosidad, la CRAD y la conductividad hidráulica muestran una variabilidad muy alta dentro de las áreas estudiadas, de modo que no es posible distinguir tendencias claras a lo largo del transecto. Las cantidades en poros gruesos y las conductividades hidráulicas son medias a altas en todas las zonas estudiadas, registrándose las más altas en Aísa. La CRAD varía dependiendo de la profundidad del suelo y de la profundidad enraizable. Aquí es donde se muestran claramente los efectos de la erosión, ya que los suelos fuertemente erosionados pueden llegar a tener capacidades de retención de agua disponible extremadamente bajas.

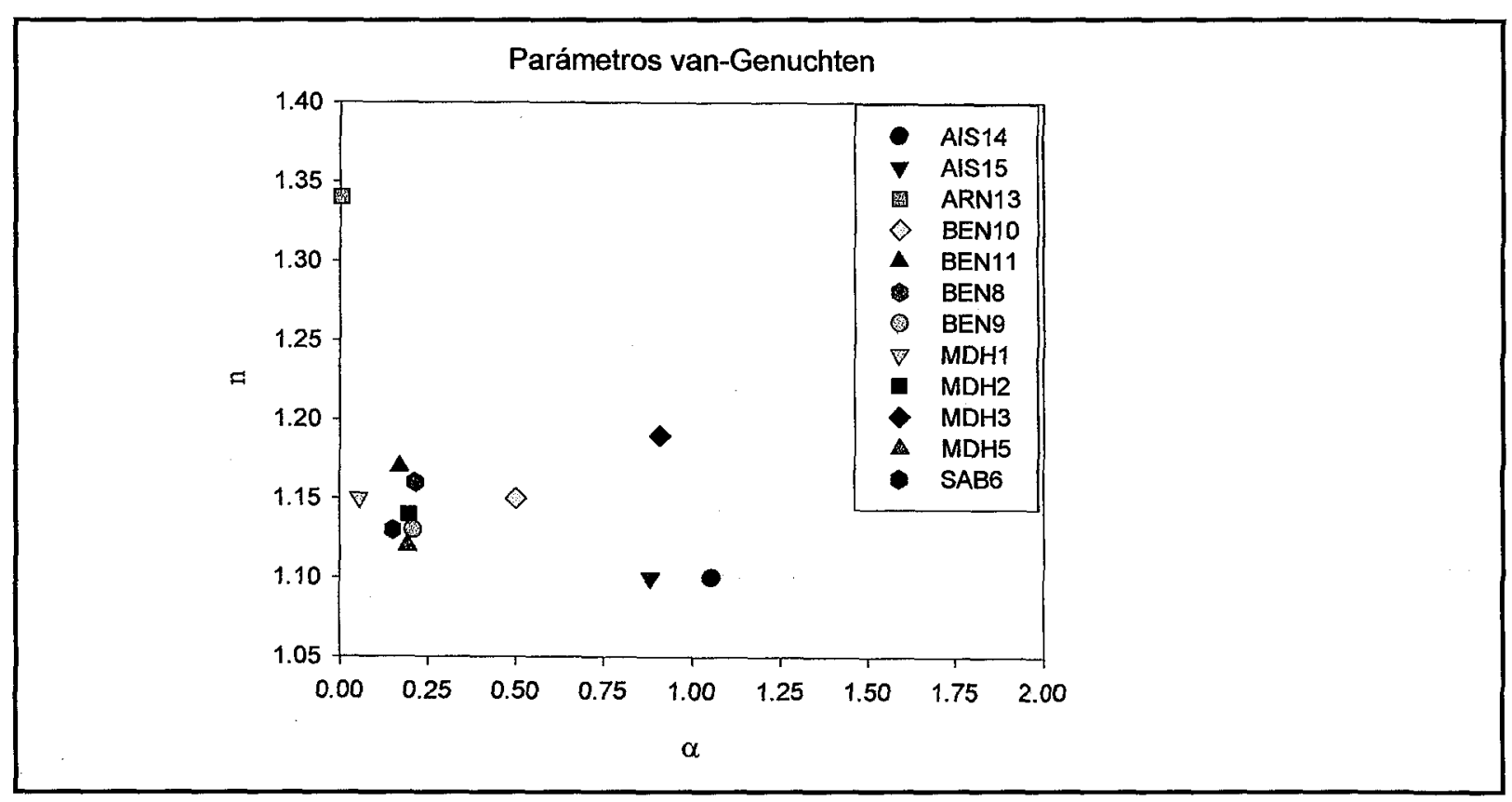

Figura 8: Parámetros van-Genuchten $(a, n)$ de los perfiles estudiados. Medias calculadas tras su estimación en cada horizonte. Se observa la similitud de la mayoria de los suelos estudiados.

Abb. 8: Berechnete van-Genuchten Parameter der untersuchte Profile. Dargestellt sind die Mittelwerte der einzelnen 
FACTORES HIIDRICOS Y EROSIONABILIDAD EN EL GRADIENTE EDAFOCLIMÁTICO

La representación de los parámetros van-Genuchten de las características hidráulicas (Figura 8) de las matrices de los suelos estudiados confirma el parecido de los factores hidráulicos de los suelos. Solamente el suelo de Arnás y los suelos de Aísa son distintos. El primero se diferencia por su alto contenido en arcillas, lo cual puede condicionar el comportamiento hidráulico; las diferencias de los suelos de Aísa se deben esencialmente al enraizamiento intenso y la subsecuente densidad aparente baja de los horizontes superficiales.

\section{Agradecimientos}

Este trabajo se ha realizado con el apoyo del proyecto EPRODESERT, financiado por la DFG/Alemania (Ri 835/1-(1-3)) bajo el titulo "Landnutzungswandel, Erosion und Desertifikation in Nordostspanien" (Cambios de uso del suelo, erosión y desertificación en el noreste de España). Además, M. Seeger está apoyado por el gobierno de Baden-Württemberg y el DAAD (Alemania) con una beca personal.

\section{Referencias bibliogrâficas}

ARBEITSGRUPPE BoDEN (Eds.) (1996): Bodenkundliche Kartieranleitung 4. Auflage, Nachdr., E. Schweizerbart'sche Verlagsbuchhandlung Stuttgart, Hannover, 392 pp.

Cernusca, A.; TAPpeiner, U.; BAHN, M. (1996): ECOMONT; ecological effects of land use changes on european terrestrial mountain ecosystems. Pirineos, 147-148, 145-172.

Cuadrat Prats, J.M. (1995): Régimen pluviométrico; Atlas de geografía de Aragón; Institución "Fernando el Católico", Zaragoza.

Deutsches Institut FÜR Normung (Din) (1973): DIN 19683, Blatt 1, April 1973:

Physikalische Laboruntersuchungen - Bestimmung der

Korngrößenzusammensetzung durch Siebung; . DIN 19683, Blatt 2, April 1973: Physikalische Laboruntersuchungen - Bestimmung der

Korngrößenzusammensetzung nach Vorbehandlung mit Natriumpyrophosphat.

Disse, M. (1995): Modellierung der Verdunstung und der Grundwasserneubildung in ebenen Einzugsgebieten, IHW, Universität Karlsruhe, 180 pp.

García Ruiz J. M.; Lasanta, T.; Ruiz Flaño, P.; Martí, C.; Ortigosa, L.; González, C. (1994): Soil Erosion and Desertification as a Consequence of Farmland Abandonment in Mountain Areas. Desertification Bulletin, 25, 27-33.

García Ruiz, J. M., LASANTA T. (Eds.) (1994): Efectos geomorfológicos del abandono de tierras. Sociedad Española de Geomorfología, Zaragoza, 171 pp.

GARCíA-RUIZ, J.M., LASANTA, T. et al. (1996): Efectos hidrológicos y geomorfológicos de la reciente expansión del matorral en el Pirineo Central español. $A P T$ Berichte, 8; 89-97. 
Ibarra Benlloch, P. \& Martínez Ferrer. J (1995): Perfiles de vegetación del Pirineo aragonés. Atlas de geografía de Aragón; Institución "Fernando el Católico", Zaragoza.

ICONA [Eds.] (1988): Agresividad de la lluvia en España-Valores del factor $R$ de la Ecuación Universal de Perdidas de Suelo. Ministerio de Agricultura, Pesca y Alimentación; Madrid.

Molinillo, M.; Lasanta, T.; García-Ruiz, J. M. (1997): Managing Mountainous Degraded Landscapes After Farmland Abandonment in the Central Spanish Pyrenees. Environmental Management, 21(4), 587-598.

Pardini, G.; Aringhieri, R.; Plana, F.; Gallart, F. (1991): Soil properties relevant to land degradation in abandoned sloping fields in Aísa Valley, Central Pyrenees (Spain). Pirineos, 137, 79-93.

Porta Casanellas, J.; López-Acevedo, M.; Roquero de Laburu, C. (1999): Edafología. Para la agricultura y el medio ambiente 2. Ed., Ediciones Mundi-Prensa, Madrid, 849 pp.

Ries, J. B.; SeEger, M.; MarzolfF, I. (1997): El proyecto EPRODESERT. Cambios de uso del suelo y morfodinámica en el nordeste de España. Geographicalia, 37, 205-225.

Saña Vilaseca, J.; Moré Ramos, J.; Cohí Ramón, A. (1996): La gestión de la fertilidad de los suelos, Ministerio de Agricultura, Pesca y Alimentación, Madrid, 277 pp. Schwertmann, U., VOGL, W. \& KAINZ, M (1987): Bodenerosion durch Wasser Vorbersage des Abtrags und Bewertung von Gegenmaßnabmen; Ulmer; Stuttgart, $64 \mathrm{pp}$.

SEEGER, M. (1997): Untersuchungen zu Wasser und Stoffhaushalt auf aufgelassenen Ackerflächen in Nordost-Spanien. APT-Berichte, 8, 52-70.

Suárez Cardona F., Sainz Ollero H., Santos Martínez T., Bernáldez F.G. (1992): las Estepas Ibéricas; Ministerio de Obras Públicas y Transportes, Centro de Publicaciones, Madrid, 160 pp.

USDA (EDs.) (1997): Keys to Soil Taxonomy, 7. Ed. Washington D.C., 326 pp.

van Genuchten, M. Th. (1980): A Closed-form Equation for Predicting the Hydraulic Conductivity of Unsaturated Soils. Soil Sci. Soc. Am. J., 44, 892-898.

WischmeIER, W.H. \& SMITH, D. (1978): Predicting rainfall erosion losses - A guide to conservation planning; USDA; Agric. Handbook 537, Washington D. C., 58 pp Young, R.A.; Römkens, M.J.M.; McCool, D.K. (1990): Temporal Variations in Soil Erodibility. Catena Supplement, 17, 41-53. 